\title{
Social behaviour in stallion groups (Equus przewalskii and Equus caballus) kept under natural and domestic conditions
}

\author{
Janne Winther Christensen ${ }^{\mathrm{a}, *}$, Tatjana Zharkikh ${ }^{\mathrm{b}}$, \\ Jan Ladewig ${ }^{\mathrm{c}}$, Natalja Yasinetskaya ${ }^{\mathrm{b}}$ \\ ${ }^{a}$ Department of Animal Health and Welfare, Danish Institute of Agricultural Sciences, \\ Research Centre Foulum, P.O. Box 50, 8830 Tjele, Denmark \\ biosphere Reserve 'Askania-Nova', Askania-Nova, Chaplinskii r-n, Khersonskaja obl. 75230, Ukraine \\ ${ }^{\mathrm{c}}$ Department of Animal Science and Animal Health, The Royal Veterinary and Agricultural University, \\ 1870 Frederiksberg $C$, Denmark
}

Accepted 8 November 2001

\begin{abstract}
The aim of this study was to investigate social behaviour in differently reared stallions in their respective environments; one group of stallions was reared under typical domestic conditions whereas the other group was reared and lives under natural conditions. The domestic group consisted of 19, 2-year-old stallions (Equus caballus), which were all weaned at 4 months of age and experienced either individual or group housing facilities before being pastured with the other similarly aged stallions. The natural living and mixed age group of Przewalski stallions $(E$. przewalskii) consisted of 13 stallions, most of which were juveniles $(n=11, \leq 4$ years; $n=2,>9$ years). The domestic group was studied in a 4-ha enclosure at the Danish Institute of Agricultural Sciences and the Przewalski group under free-ranging conditions in a 75-ha enclosure in the Askania Nova Biosphere Reserve, Ukraine. Behavioural data was collected during $168 \mathrm{~h}$ of direct observation. The occurrence of 14 types of social interactions was recorded and group spacing behaviour was studied using nearest neighbour recordings. In spite of very different environments, reflecting domestic and natural rearing conditions, many similarities in behaviour was found. Play and play fight behaviour was very similar in the two stallion groups. Quantitative differences were found in social grooming since Przewalski stallions groomed more frequently $(P=0.004)$, and in investigative behaviours, since domestic stallions showed more nasal $(P=0.005)$ and body sniffing $(P<0.001)$, whereas Przewalski stallions directed more sniffing towards the genital region $(P<0.001)$. These differences may, however, be attributed to environmental factors and in the period of time the stallions were together prior to the study
\end{abstract}

\footnotetext{
*Corresponding author. Tel.: +45-89991330; fax: +45-89991500.

E-mail address: jannewinther.christensen@agrsci.dk (J.W. Christensen).
} 
period. Quantitative differences appeared in some agonistic behaviours (kick threat, $P<0.001$; and kick, $P<0.001$ ), but data do not support earlier findings of Przewalski horses being significantly more aggressive than domestic horses. In general, Przewalski stallions engaged in more social interactions, and they showed less group spacing, i.e. maintained a significantly shorter distance between neighbours $(P<0.001)$. The study indicates that also domestic horses, which have been reared under typical domestic conditions and allowed a period on pasture, show social behaviour, which is very similar to that shown by their non-domestic relatives. (C) 2002 Elsevier Science B.V. All rights reserved.

Keywords: Domestic horse; Przewalski horse; Stallion group; Social behaviour; Equus caballus; Equus przewalskii

\section{Introduction}

Animals living under natural conditions are regarded as showing the normal behaviour of that species, and deviations from normal behaviour in domestic animals may relate to changes resulting from domestication, or they may be caused by environmental factors. Price (1984) defined domestication as 'that process by which a population of animals becomes adapted to man and to the captive environment by some combination of genetic changes occurring over generations and environmentally induced developmental events reoccurring during each generation'. Domestic animals generally show reduced responsiveness to environmental changes, which may not only be expressed in their reaction towards humans, but also in intra-specific reactions and investigative behaviours. Domestication may additionally induce a change in group spacing behaviour since management practices, which minimise competition for resources, should permit captive populations to exist at relatively high densities (Price, 1984).

Studies of feral horses living under natural conditions indicate that the species-specific behaviour of equids has remained relatively unaffected by the domestication process (Tyler, 1972; Feist and McCullough, 1976; Waring, 1983), and thus domestication seems to have influenced the quantitative rather than the qualitative nature of social behaviour in horses. Quantitative changes may include a reduction in aggressive behaviour because the domestic environment reduces the selective advantages of aggressive potential in securing resources.

Direct comparisons of social behaviour in domestic and non-domestic horses are lacking in the literature, and are made difficult by the fact that domestic horses are kept under conditions, which are very different to the natural environment. Typical rearing conditions for domestic horses include early weaning of foals, commonly at the age of 5-6 months, after which they may experience either individual housing conditions or enter groups with other similarly aged horses. In sharp contrast to these conditions, studies on horses in natural environments show that horses tend to live in mixed age groups and stay with their natal herd till approximately 2 years of age (for a review see Waring, 1983). The rearing environment and the environment in which an animal lives is likely to affect the behaviour of the individual. For instance, it has been shown that social restrictions affect the behaviour of horses (Christensen et al., 2002), and rearing animals in physical isolation from older, socially dominating conspecifics may preclude the development of normal 
adult-like behaviour patterns of agonistic behaviours and may result in the retention of juvenile social behaviours (Price, 1984).

Feh (1988) and Keiper and Receveur (1992) reported more aggressive behaviour in groups of Przewalski horses, which is the closest wild relative of domestic horses, than in feral horse groups. However, these findings were based upon a limited number of stallions (three Przewalski stallions versus eight Camargue stallions by Feh, 1988), or upon comparisons between different studies (Keiper and Receveur, 1992). The present study aims to extend these earlier investigations by studying social behaviour in Przewalski and domestic stallions within the same study, with a large number of stallions per group, and using the same observational techniques and observers on both groups.

Investigations on behavioural differences between domestic horses and their wild relatives-displayed in their respective home environments-are of great importance in understanding the span of equid behaviour. The present study investigates and discusses social and group spacing behaviour in domestic stallions and in non-domestic Przewalski stallions, reared under typical domestic and natural conditions, respectively.

\section{Materials and methods}

\subsection{Animals}

The domestic group consisted of 19 2-year-old stallions, reared under typical domestic conditions at the Danish Institute of Agricultural Sciences. The stallions were weaned at approximately 4 months of age and housed either individually $(n=7)$ or in groups of three $(n=12)$ with daily access to paddocks during the autumn, winter and spring season and were pastured together during the summer season. The stallions were pastured in two separate groups ( $n=7$ and $n=12$ ), which were joined into one group of 19 stallions after 7 weeks on pasture. The study period began 5 days after formation of the joined group, allowing for dominance relationships to get settled, which normally happens within a few days (Waring, 1983; Tilson et al., 1988).

The Przewalski group consisted of 13 stallions, five 2-year-old, two 3-year-old, four 4year-old and two older experienced breeding stallions aged 9 and 13 years, respectively. The stallions belong to the Askania Nova Biosphere Reserve, Ukraine, in which horses are bred under natural conditions in large enclosures (up to 1200 ha.). Foals are captured, freeze branded and ear notched in order to aid the recognition of individuals. Young stallions remain in their natal herd until approximately 2 years of age, which is the usual age of dispersal of stallions under natural conditions (Khalil and Kaseda, 1997). Surplus stallions, i.e. stallions without a harem, are kept in separate enclosures, and the observed stallion group was formed approximately 1 month prior to the study period. The 2-year-old stallions came from their natal herd, whereas, the other stallions previously were part of another stallion band.

There were no mares present in the vicinity of either of the two stallion groups, nor did the stallions have to compete for resources. Grass was abundant and evenly distributed and there was free access to water. The size of the enclosures differed, as the domestic group was kept in a 4-ha enclosure, whereas, the Przewalski group had access to 75 ha. 
Table 1

Ethogram of recorded behaviours

\begin{tabular}{|c|c|}
\hline Behaviour & Description $^{\mathrm{a}}$ \\
\hline Displacement & $\begin{array}{l}\text { Approach of one horse with ears back causes another to move away so that distance } \\
\text { is maintained or increased, without overt aggression }\end{array}$ \\
\hline Mouth clapping & $\begin{array}{l}\text { Submissive behaviour. Opening and closing mouth with lips retracted. Typically, the } \\
\text { head and neck are extended, and the ears oriented back or laterally. By some termed } \\
\text { snapping }\end{array}$ \\
\hline Threat to bite & Bite intention movement with ears back and neck extended, with no actual contact \\
\hline Threat to kick & $\begin{array}{l}\text { Kick intention movement, performed by swinging rump or backing up, or by waving } \\
\text { or stamping hindleg toward another horse, without making contact }\end{array}$ \\
\hline Bite & $\begin{array}{l}\text { Opening and rapid closing of the jaws with actual contact to another horse's body. } \\
\text { The ears are back and lips retracted }\end{array}$ \\
\hline Kick & $\begin{array}{l}\text { One or both hindlegs lift off the ground and rapidly extend backwards toward } \\
\text { another horse, with apparent intent to make contact }\end{array}$ \\
\hline Push & $\begin{array}{l}\text { Pressing of the head, neck, shoulder, chest, or body against another horse, causing it } \\
\text { to move one or more legs to regain balance }\end{array}$ \\
\hline Play & $\begin{array}{l}\text { Play directed at another individual, which may or may not reciprocate; includes low } \\
\text { intensity play movements such as nipping, grasping, and pulling mane or tail }\end{array}$ \\
\hline Play fight & $\begin{array}{l}\text { High intensity play, which is reciprocated by one or more partners; includes } \\
\text { vigorous play movements such as rearing, boxing, circling, kneeling, and chasing }\end{array}$ \\
\hline Social grooming & $\begin{array}{l}\text { Reciprocal coat care in which the partners stand beside one another, usually head-to- } \\
\text { shoulder or head-to-tail, grooming each other's neck, mane, rump, or tail by gently } \\
\text { nipping, nuzzling, or rubbing }\end{array}$ \\
\hline Mounting & $\begin{array}{l}\text { One stallion raises his chest and forelegs onto another horse's back with the forelegs } \\
\text { on either side, just as during copulation, with or without erection }\end{array}$ \\
\hline Nasal sniff & Olfactory investigation. Two or more horses sniff mutually head-to-head \\
\hline Body sniff & $\begin{array}{l}\text { Olfactory investigation. A horse sniffs the neck, withers, flank, or tail of another } \\
\text { horse, which may or may not reciprocate }\end{array}$ \\
\hline Genital sniff & $\begin{array}{l}\text { Olfactory investigation. A horse sniffs the genital region of another horse, which } \\
\text { may or may not reciprocate }\end{array}$ \\
\hline
\end{tabular}

${ }^{\text {a }}$ Based on descriptions in Boyd and Houpt (1994) and McDonnell and Haviland (1995).

\subsection{Data collection}

Behavioural data was collected during July 1999 on the domestic group and during April-May 2000 on the Przewalski group. The seasons corresponded as Ukrainian spring weather and temperature corresponds to the Danish summer. Behavioural data was collected by direct observation with separate recordings of social interactions (Table 1) ( $72 \mathrm{~h}$ per group) and nearest neighbours ( $12 \mathrm{~h}$ per group; 72 recordings per horse). Observation periods lasted for 3 or $4 \mathrm{~h}$, and were equally distributed between sunrise and sunset.

Group spacing behaviour was studied by focal sampling, in which the nearest (1st neighbour) and 2nd nearest horse (2nd neighbour) of each horse were recorded every $10 \mathrm{~min}$, in total 72 recordings of neighbours for each horse. The 1st neighbour of a particular horse was defined as the horse with any part of its body closer than any other animal to the head of the focal animal. The 2 nd neighbour was likewise the 2 nd nearest horse. The distance between neighbours was estimated as within one horselength $(<1.5 \mathrm{~m})$, 
more than one horselength but less than $5 \mathrm{~m}(1.5-5 \mathrm{~m})$, or more than $5 \mathrm{~m}$ away $(>5 \mathrm{~m})$. The recording interval of 10 min was chosen in accordance with results from Wells and Feh (unpublished, quoted by Feh, 1988) showing that the probability of having the same nearest neighbour drops drastically after $8 \mathrm{~min}$. An interval of $10 \mathrm{~min}$ should therefore guarantee a certain independence of the samples (Feh, 1988).

Observations on the domestic group took place from an observation caravan situated just outside the enclosure, from which the horses were easily recognised by dye marks, coat colour, size and individual marks. At Askania Nova observations took place from a horse carriage, pulled by a trained, domestic horse. The Przewalski horses are accustomed to carriages being the normal means of transportation of people within the enclosures. Furthermore, stallions were allowed to get additionally accustomed to the presence of the carriage and observers during preliminary studies. A distance of 20-40 m was maintained and identification of horses was based on freeze brands, ear notches, coat colour, size, general shape and appearance of mane and tail.

\subsection{Data analysis}

Data on social interactions was analysed by two group comparisons by use of the $t$-test and, in cases where data failed the normal distribution, the Mann-Whitney $U$ (MWU) test was applied. Frequencies of neighbours within each distance category in the two stallion groups were likewise compared by use of the MWU test, whereas neighbour associations within groups were tested in the Wilcoxon Signed Rank (WSR) test (Siegel and Castellan, 1988). Calculations were made in the computer software SAS 6.12 (SAS Institute, 1996) and SigmaStat 2.0 (SPSS Inc., 1997). Abbreviations are given for non-parametric test results, $t$-values are given with degrees of freedom, $t(\mathrm{df})$, and a significance level of $P<0.05$ is applied.

\section{Results}

Table 2 shows the occurrence of the recorded social interactions in the two stallion groups. The naturally reared Przewalski stallions tended to show more agonistic interactions than the domestically reared stallions (Table 2), significant only for kick threats $(t(30)=3.54$; $P=0.001$ ) and kicks (MWU, $P=0.001$ ). The domestic stallions showed significantly more pushing behaviour (MWU, $P=0.016$ ), although the occurrence was very low in both groups. They likewise tended to show more mouth clapping (MWU, $P=0.055$ ), whereas Przewalski stallions tended to show more displacements (MWU, $P=0.057$ ).

Significant differences were found in investigative behaviour in the two groups of stallions. The domestic stallions showed more investigative behaviour (153.2 recordings per horse) than did the Przewalski stallions (92.8 recordings per horse, $t(30)=4.28$; $P<0.001)$. Significantly more nasal $(t(30)=3.06 ; P=0.005)$ and body sniffing (MWU, $P<0.001)$ was recorded in the domestic group, whereas Przewalski stallions directed significantly more sniffing to the genital region (MWU, $P<0.001$ ).

A significantly higher level of social grooming was recorded in the Przewalski group (0.53 per animal per hour versus domestic: 0.24 per animal per hour, MWU, $P=0.004$ ). 
Table 2

Occurrence of behaviours (mean \pm S.E.) by group

\begin{tabular}{lccc}
\hline & Domestic stallions $(n=19)$ & Przewalski stallions $(n=13)$ & $P$-value \\
\hline Behaviour per $72 \mathrm{~h}$ & & & \\
$\quad$ Displacement & $16.0 \pm 12.9$ & $26.6 \pm 9.1$ & 0.057 \\
Mouth clapping & $8.7 \pm 3.0$ & $1.0 \pm 0.5$ & 0.055 \\
Bite threat & $11.5 \pm 1.9$ & $27.1 \pm 7.8$ & 0.103 \\
Kick threat & $5.5 \pm 0.9$ & $15.4 \pm 3.1$ & $0.001^{* *}$ \\
Bite & $9.1 \pm 1.4$ & $26.8 \pm 10.4$ & 0.066 \\
Kick & $3.6 \pm 0.7$ & $9.3 \pm 1.7$ & $0.001^{* *}$ \\
Push & $1.9 \pm 0.5$ & $0.5 \pm 0.3$ & $0.016^{*}$ \\
Play fight & $39.7 \pm 5.2$ & $47.2 \pm 9.1$ & 0.527 \\
Social grooming & $17.3 \pm 5.4$ & $38.3 \pm 7.4$ & $0.004^{* *}$ \\
Play & $40.6 \pm 4.13$ & $57.5 \pm 8.6$ & 0.060 \\
Mounting & $1.2 \pm 0.6$ & $0.8 \pm 0.4$ & 0.743 \\
Nasal sniff & $88.5 \pm 5.1$ & $65.5 \pm 5.3$ & $0.005^{* *}$ \\
Body sniff & $61.2 \pm 6.4$ & $11.8 \pm 1.5$ & $<0.001^{* *}$ \\
Genital sniff & $3.5 \pm 0.7$ & $15.5 \pm 3.7$ & $<0.001^{* *}$ \\
\hline
\end{tabular}

* Significant difference at $P<0.05$.

** Significant difference at $P<0.01$.

Likewise, the Przewalski stallions tended to show more play behaviour $(t(30)=1.95$; $P=0.060$ ), whereas, no difference was found in the level of play fighting (MWU, $P=0.527)$. In the domestic group, $49 \%$ of all playful events were higher intensity play fighting, whereas, play fighting accounted for $45 \%$ of all playful events in Przewalski stallions. The occurrence of mounting behaviour was rather low and not significantly different between the two groups.

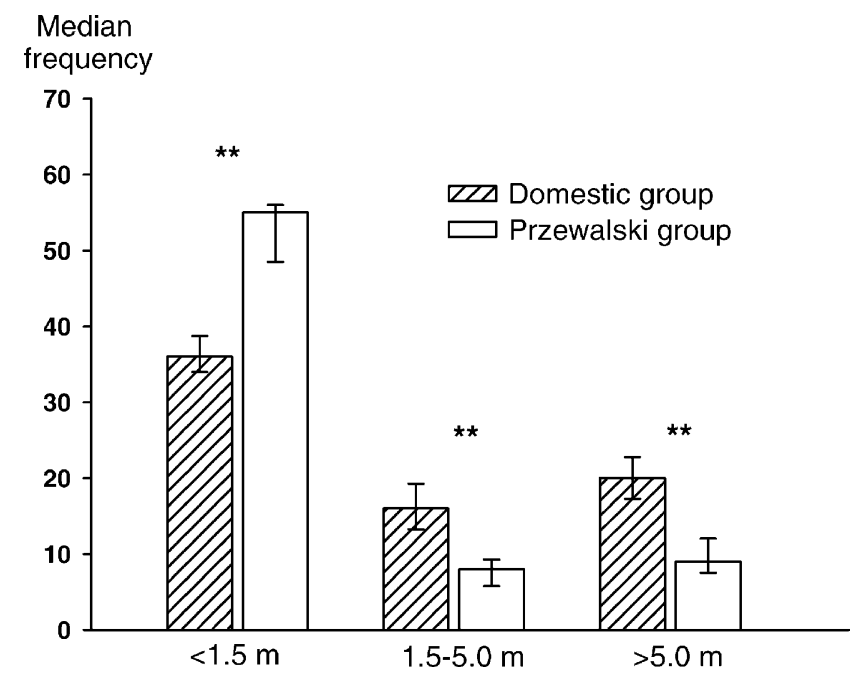

Fig. 1. Distance between nearest neighbours (1st neighbours) in domestic and Przewalski stallions. Medians and $25-75 \%$ quartiles are given $(* *$ significant difference at $P<0.01)$. 
The Przewalski stallions had a tighter group structure than did the domestic stallions as the distance between nearest neighbours was significantly shorter in the Przewalski group than in the domestic group for both 1st (MWU, $P<0.001$ ) (Fig. 1) and 2nd neighbours (MWU, $P<0.001$ ).

\section{Discussion}

A higher number of social interactions was recorded in the naturally reared and mixed age group of Przewalski stallions than in the domestically reared and similarly aged group of domestic stallions. Consistently, a significantly higher level of social grooming was recorded in the Przewalski group. Other authors have found that levels of social grooming vary with season, with peaks during the spring period, due to the shedding of the winter coat (Tyler, 1972; Kimura, 1998; Zharkikh, 2000). The Przewalski horses at Askania Nova grow a thick winter coat due to low winter temperatures, and the stallions were therefore still shedding their winter coats during the observations, which may be the main reason for the high occurrence of grooming behaviour. Furthermore, the insect pressure on the Przewalski group seemed to be higher than on the domestic group, and insects may contribute to itching of the coat. Therefore, the difference in occurrence of social grooming should probably be ascribed to environmental conditions.

The Przewalski group also tended to be more playful than the domestic group (on average 1.5 versus 1.1 playful events per horse per hour). Juveniles play when their primary needs are met and the performance of play has been suggested to be an indicator of good welfare in wild as well as captive juveniles (Fagen, 1981). The high occurrence of play behaviour in both groups therefore indicates good conditions for both groups of horses. The proportion of playful events being play fight behaviour, which is believed to be of importance for both the social and the physical development, did not differ in the two stallion groups.

Przewalski stallions showed significantly more rear directed interactions (threats to kick and kick) which may be used both in a defensive and offensive context (Feh, 1988; Dierendonck et al., 1995). Thus, Przewalski stallions tended to show more aggressive behaviour than domestic stallions, which supports the findings of Feh (1988) and Keiper and Receveur (1992). Food and water was abundant in both enclosures and no mares were present in the vicinity of either of the stallion groups and thus the stallions did not have to compete for resources. The higher number of aggressions may, however, relate to the fact that the two adult stallions were present in the Przewalski group as it has been shown that adults are more aggressive than juveniles (Feh, 1988). It should also be taken into consideration that Przewalski stallions also engaged in more friendly interactions, such as social grooming and play. Thus, the Przewalski stallions of the present study merely seems to more socially active, and thus data do not support earlier findings about E. przewalskii being significantly more aggressive than E. caballus.

Feral horses in natural habitats have been observed to form groups of two to more than 20 individuals, with the most common group size being four or five individuals (reviewed by Waring, 1983). Group size probably affects social behaviour, and interactions in a smaller group may be different from interactions in a larger group. Feh (1988) 
reported an aggression rate of 0.76 per horse per hour in a small $(n=4)$ Przewalski bachelor group, which is only half the rate observed in Przewalski stallions in the present study $(1.46, n=13)$. This difference may be caused by the different group sizes. In the present study, both groups were rather large and both exceeded the most commonly observed group size.

Living conditions of the two stallion groups reflect natural and typical domestic conditions, respectively. The domestic stallions experienced early weaning, individual or group housing facilities and semi free conditions on pasture during the summer period with similarly aged conspecifics. The Przewalski group, on the other hand, experienced natural conditions and stayed with their natal herd until the age of 2 years and subsequently joined a mixed age stallion group in a 75-ha enclosure. Boyd (1988) studied ontogeny of behaviour in Przewalski horses and compared it to other studies on ontogeny in domestic horses and concluded that the time line for onset of behaviours was very similar to those reported for domestic horses. Domestically kept horse groups normally differ from natural horse groups in age structure since domestics are typically kept with other similarly aged horses, whereas natural groups are of mixed ages. Dominance relationships within groups typically depends on physical characteristics, age, experience, and length of residency of the herd (reviewed by Boyd and Houpt, 1994), and the two adult and experienced breeding stallions in the Przewalski group were clearly dominant to all juveniles in the group. In the domestic group, however, a few individuals were also clearly dominant to all others, and thus clear dominance hierarchies existed in both groups. Dominant individuals affect the behaviour of subordinate individuals, and the presence of the adult stallions in the Przewalski group may not have affected social interactions within the group differently than did the dominant individuals of the domestic group.

There was a tendency towards Przewalski stallions making more use of displacements, whereas domestic stallions tended to show more mouth clapping, which is used by inferior individuals to express submission. Although other authors studying the formation of bachelor groups reported that a distinct social structure emerged within the 1st day after introduction (Tilson et al., 1988) the fact that the domestic group was more recently formed than the Przewalski group ( 5 days versus 1 month) probably contributed to this difference along with the different age structures. Houpt and Keiper (1982) assign a high level of displacements to a stable group structure, in which all individuals know their place; whereas mouth clapping, which is shown only by immature animals, may be used as an appeasement gesture to avoid aggression from higher ranking individuals (Tyler, 1972; Waring, 1983). Investigative behaviour was recorded more frequently in the domestic group, which again may be attributed to the later formation of their group. A different investigative pattern was evident in the two groups, as domestic horses showed more nasal and body sniffing whereas Przewalski horses directed more sniffing towards the genital region. Genital investigation may be more important to the older and more sexually mature Przewalski stalllions.

Studies of wild and feral equids have shown that stallions rarely hurt each other even in real combat, due to a well-developed social language and ritualised signals, which make inferior animals retreat to avoid the risks and costs of a fight, they are unlikely to win anyway (Klingel, 1967; Tilson et al., 1988; McDonnell and Haviland, 1995). Under domestic conditions, however, stallions are typically separated to avoid aggression and 
injury. Aggression may be induced by limited resources, the presence of mares, space restrictions, or by a lack of dealing with social encounters. In the present study, no stallions were observed to get injured in interactions with other stallions in neither of the two stallion groups. In the Przewalski group, adult stallions with breeding experience lived successfully in a bachelor group with other stallions. Davidson (1999) notes that when living in a herd, conflicts are typically avoided by spatial distribution since the horses spread out and allow only a few individuals to be within their personal space. In the present study, however, the Przewalski stallions did not spread out on their large pasture, but grouped up tightly. Thus group spacing behaviour differed in Przewalski and domestic stallions, since Przewalski stallions stayed significantly closer together, i.e. maintained a significantly shorter distance to both 1 st and 2 nd neighbours, than did domestic stallions. The domestic group was often observed to subdivide into grazing units of two or three individuals, whereas, the Przewalski group rarely subdivided. Other authors have found environmental influence on group spacing behaviour, for instance Tyler (1972) reported that on warm summer days New Forest ponies tended to gather in the shade, and at these times grooming rates peaked as well. Rutberg (1987) studied the influence of horse fly harassment on group spacing behaviour, and found that feral Assategue ponies responded to fly harassment by gathering into tighter groups. In the present study, insect pressure may be a cause of the tighter group structure in Przewalski stallions. There is probably a strong coherence between the tighter group structure of Przewalski stallions and the increased level of interactions, since horses which stay close together are more likely to interact and vice versa.

\section{Conclusions}

The qualitative nature of social behaviours was alike in domestic and Przewalski stallions, and the same behavioural definitions could be applied to register social behaviour in both groups. Play and play fight behaviour was both qualitatively and quantitatively very similar in the two stallion groups. Quantitative differences were found in social grooming and in investigative behaviours but these may be attributed to environmental factors and in the time since group formation. Quantitative differences were also evident in some agonistic behaviours but, in contrast to hypotheses of reduced aggression as a consequence of domestication, our data do not support earlier findings of Przewalski horses being significantly more aggressive than domestic horses. The study indicates that also domestic horses, which have been reared under typical domestic conditions and allowed a period on pasture, show social behaviour, which is very similar to that shown by the non-domestic Przewalski horses roaming freely on the flat steppe of Ukraine.

\section{Acknowledgements}

Thanks to Jens Malmkvist and Eva Sondergaard, Department of Animal Health and Welfare, DIAS, for comments on earlier versions of the manuscript, and to Erik L. Decker, DIAS, for technical assistance. Thanks to Julia Alexeyeva and Alla Nikitina, Kyiv State Zoological Park and to Helen Kraevoj, Biosphere Reserve Askania-Nova. 


\section{References}

Boyd, L.E., 1988. Ontogeny of behavior in Przewalski horses. Appl. Anim. Behav. Sci. 21, 41-69.

Boyd, L.E., Houpt, K.A., 1994. Activity patterns. In: Boyd, L., Houpt, K.A. (Eds.), Przewalski's Horse: the history and biology of an endangered species. State University of New York Press, Albany, pp. 195-254.

Christensen, J.W., Ladewig, J., Sondergaard, E., Malmkvist, J., 2002. Effects of individual vs. group stabling on social behaviour in domestic stallions. Appl. Anim. Behav. Sci. 75, 233-248.

Davidson, H.P.B., 1999. Natural horse-unnatural behaviour: why understanding natural horse behaviour is important. In: Proceedings of the BEVA Specialist Days on Behaviour and Nutrition, September 1999, Harrogate, UK.

Dierendonck, M.C.V., De Vries, H., Schilder, M.B.H., 1995. An analysis of dominance, its behavioural parameters and possible determinants in a herd of Icelandic horses in captivity. Netherlands J. Zool. 45, 362 385.

Fagen, R.M., 1981. Animal Play Behaviour. Oxford University Press, New York, 684 pp.

Feh, C., 1988. Social behaviour and relationships of Przewalski horses in Dutch semi-reserves. Appl. Anim. Behav. Sci. 21, 71-87.

Feist, J.D., McCullough, D.R., 1976. Behavior patterns and communication in feral horses. Z. Tierpsychol. 41, 337-371.

Houpt, K.A., Keiper, R., 1982. The position of the stallion in the equine dominance hierarchy of feral and domestic ponies. J. Anim. Sci. 54, 945-950.

Keiper, R., Receveur, H., 1992. Social interactions of free-ranging Przewalski horses in semi-reserves in The Netherlands. Appl. Anim. Behav. Sci. 33, 303-318.

Khalil, A.M., Kaseda, Y., 1997. Behavioral patterns and proximate reason of young male separation in Misaki feral horses. Appl. Anim. Behav. Sci. 54, 281-290.

Kimura, R., 1998. Mutual grooming and preferred associate relationships in a band of free-ranging horses. Appl. Anim. Behav. Sci. 59, 265-276.

Klingel, H., 1967. Soziale Organisation und Verhalten freilebender Steppenzebras. Z. Tierpsychol. 24, 580-624.

McDonnell, S.M., Haviland, J.C.S., 1995. Agonistic ethogram of the equid bachelor band. Appl. Anim. Behav. Sci. 43, 147-188.

Price, E.O., 1984. Behavioural aspects of domestication. Q. Rev. Biol. 55, 1-32.

Rutberg, A.T., 1987. Horse fly harassment and the social behavior of feral ponies. Ethology 75, 145-154.

SAS Institute, 1996. SAS Users Guide, Statistics Version 6, 4th Edition. Statistical Analysis Systems Institute, Cary, NC.

Siegel, S., Castellan, Jr., N.J., 1988. Nonparametric statistics for the behavioural sciences, 2nd Edition. McGrawHill, NY, 399 pp.

SPSS Inc., 1997. SigmaStat, Statistical Software, version 2.0 for Windows 95, User's Manual. SPSS Inc., USA.

Tilson, R.L., Sweeny, K.A., Binczik, G.A., Reindl, N.J., 1988. Buddies and bullies: social structure of a bachelor group of Przewalski horses. Appl. Anim. Behav. Sci. 21, 169-185.

Tyler, S.J., 1972. The behaviour and social organisation of the New Forest ponies. Anim. Behav. Monogr. 5-6, 85-196.

Waring, G.H., 1983. Horse Behaviour: the behavioural traits and adaptations of domestic and wild horses, including ponies. Noyes, Park Ridge, NJ, 292 pp.

Zharkikh, T.L., 2000. Comfort behaviour of Przewalski horses (E. przewalskii) at the Biosphere Reserve "Askania Nova". In: Havrylenko, V.S. (Ed.), News Biosphere Reserve 'Askania Nova': protection and preservation of rare species. Askania Nova, Ukraine, pp. 40-49. 